\title{
On the mechanism of ionization oscillations in Hall thrusters
}

\author{
O. Chapurin, ${ }^{1, a)}$ A. I. Smolyakov, ${ }^{1}$ G. Hagelaar, ${ }^{2}$ and Y. Raitses ${ }^{3}$ \\ 1) Department of Physics and Engineering Physics, University of Saskatchewan, \\ Saskatoon SK S7N 5E2, Canada \\ 2) LAPLACE, Université de Toulouse, CNRS, INPT, UPS, 118 Route de Narbonne, \\ 31062 Toulouse, France \\ 3) Princeton Plasma Physics Laboratory, Princeton, New Jersey 08540, \\ $U S A$
}

Low frequency ionization oscillations involving plasma and neutral density (breathing modes) are the most violent perturbations in Hall thrusters for electric propulsion. Because of its simplicity, the zero-dimensional (0-D) predator-prey model of two nonlinearly coupled ordinary differential equations for plasma and neutral density has been often used for the characterization of such oscillations and scaling estimates. We investigate the properties of its continuum analog, the one-dimensional (1-D) system of two nonlinearly coupled equations in partial derivatives (PDE) for plasma and neutral density. This is a more general model, of which the standard 0-D predatorprey model is a special limit case. We show that the 1-D model is stable and does not show any oscillations for the boundary conditions relevant to Hall thruster and the uniform ion velocity. We then propose a reduced 1-D model based on two coupled PDE for plasma and neutral densities that is unstable and exhibit oscillations if the ion velocity profile with the near the anode back-flow (toward the anode) region is used. Comparisons of the reduced model with the predictions of the full model that takes into account the self-consistent plasma response show that the main properties of the breathing mode are well captured. In particular, it is shown that the frequency of the breathing mode oscillations is weakly dependent on the final ion velocity but shows a strong correlation with the width of the ion back-flow region.

a)Electronic mail: alex.chapurin@usask.ca 


\section{INTRODUCTION}

Hall thrusters are electric propulsion plasma devices with cross-field $E \times B$ configuration, where electrons are effectively trapped by the magnetic field, and heavy ions are accelerated by the electric field generating thrust. Despite the long history and a large number of successful missions, as well as the relatively simple design of these devices, many physical phenomena are not well understood. Large amplitude discharge current oscillations in the axial direction of Hall thrusters, or the so-called breathing modes, are among the most strong perturbations that affect the operation, e.g. with current oscillations reaching $100 \%$ and even extinguishing the discharge ${ }^{1}$. However note that some modifications of Hall thrusters exhibit no or very little low-frequency oscillations ${ }^{2 \mid 3}$. The physics of the breathing mode has been studied in many papers but the full understanding is far from complete. Simulations of low-frequency axial dynamics of Hall thrusters were done earlier with various approxima-

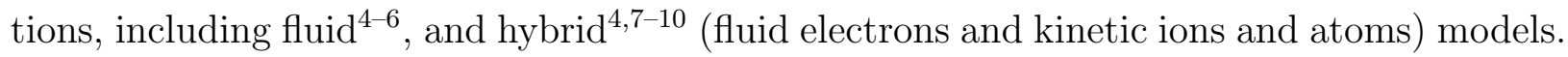
Ionization is one of the key physics elements of the breathing mode oscillations. In addition to the basic coupling of plasma density and neutral gas due to the ionization, many models include self-consistent electric field calculations along the channel, and some models include electron pressure evolution and detailed electron energy balance.

At the same time, a simple zero-dimensional (0-D) model was proposed ${ }^{11112}$ to explain the mechanism of breathing mode, in which neutral and ion (plasma) densities are coupled in the form of predator-prey model (Lotka-Volterra equations). The predator-prey model is a system of two nonlinearly coupled ordinary differential equations that describes periodic oscillations in a number of physics and biology systems. This model is claimed to predict the frequencies close to the observed in experiments. It was, however, noted that for the constant ion and neutral velocities, the model is stable and does not predict any instability 13 so the conditions for the excitation of the ionization oscillations are not clear. Additional effects and modifications ${ }^{13[14}$ were proposed for the predator-prey model to make it unstable but it remains unclear to what degree the 0-D models are capable to predict the breathing mode oscillations (discussed in section II).

Nevertheless, simple reduced models are of considerable interest. First, such models are useful as scaling tools, e.g. for the design of power supply sources. Another motivation stems from the considerable complexity of the full models. In the full models with many parameters 
that are unknown experimentally, it is rather difficult to predict the conditions for the instability and therefore difficult to translate the predictions of the theoretical models into practical recommendations. The search for physical mechanism (or mechanisms) and critical controlling parameters continues ${ }^{15}$. Reduced models focusing on selected specific phenomena may allow easier experimental validation and eventually lead to a better understanding of the physics of the instability, its conditions, and the development of the full predictive $\operatorname{model}(\mathrm{s}) \underline{16 \mid 17}$.

In this work, we analyze the properties of the continuum one-dimensional (1-D) model, based on two coupled partial differential equations (PDE) for the evolution of neutral and plasma densities. The 0-D predator-prey is a reduction of the 1-D model. If anything, the more complete 1-D model should exhibit the same physics (and e.g. predict similar characteristics of the oscillations) if the reduction from the 1-D to the $0-\mathrm{D}$ model is appropriate for the relevant physics, and the oscillations in the 0-D model are not the artefact introduced by the simplifications. We show, however, that the 1-D model is not only stable with boundary conditions of a typical Hall thruster and the standard assumptions of the uniform velocity (as it is used in 0-D model) but also does not show the oscillations observed in the 0-D model. On the contrary, any oscillations, even introduced externally, are damped in time and space. We then propose a modified 1D model with a non-uniform ion velocity profile and with a back-flow (toward the anode) the region near the anode. We show that such a model is unstable and exhibit ionization oscillations with characteristics roughly consistent with the predictions of the full fluid model 18 . Rather good agreement of the results of the reduced (simplified) and full models suggests that the reduced model captures well essential physics relevant to the breathing mode and may point to the critical conditions for the instability.

The paper is organized as follows. In section II, various modifications of the 0-D model are discussed. In section III, the continuum 1-D model is introduced. The stationary solutions are obtained here, their stability and response to external perturbations are discussed for the uniform profile of the ion velocity. Section $\mathrm{IV}$ presents a reduced 1-D continuum model with the ion back-flow region near the anode and shows that this model exhibit unstable nonlinear oscillations. In section $\mathrm{V}$, the predictions of the reduced model are compared against the full self-consistent simulations. 


\section{ZERO-DIMENSIONAL (0-D) PREDATOR-PREY MODELS}

In this section we discuss the various modifications and properties of the zero-dimensional (0-D) predator-prey models for the ionization oscillations. Such a simplest model includes basic time and space dynamics of plasma and neutrals and is based on the continuity equations for the ion density $n_{i}$ (assuming full plasma quasineutrality), and neutral atom density $n_{a}$ :

$$
\begin{aligned}
& \frac{\partial n_{a}}{\partial t}+v_{a} \frac{\partial n_{a}}{\partial x}=-\beta n_{a} n_{i}, \\
& \frac{\partial n_{i}}{\partial t}+\frac{\partial}{\partial x}\left(n_{i} v_{i}\right)=\beta n_{a} n_{i},
\end{aligned}
$$

where $v_{i}, v_{a}$ are ion and atom flow velocities, respectively, and $\beta$ is the ionization rate coefficient. We assume that $v_{a}$ is a constant, but $v_{i}$ and $\beta$, in general, could be non-uniform in space. A basic assumption leading to a simple zero-dimensional predator-prey mode 11 is that the ionization zone and acceleration zone is replaced by the transition layer of the width $L$. The integration of Eqs. (1)-(2) over this region results in the system of ordinary differential equations (ODE):

$$
\begin{aligned}
& \frac{\partial}{\partial t}\left\langle n_{a}\right\rangle+\left.\frac{1}{L}\left(n_{a} v_{a}\right)\right|_{0} ^{L}=-\beta\left\langle n_{a} n_{i}\right\rangle, \\
& \frac{\partial}{\partial t}\left\langle n_{i}\right\rangle+\left.\frac{1}{L}\left(n_{i} v_{i}\right)\right|_{0} ^{L}=\beta\left\langle n_{a} n_{i}\right\rangle,
\end{aligned}
$$

where averages over the transition layer are defined as $\langle(\ldots)\rangle=L^{-1} \int_{0}^{L}(\ldots) d x$ and $\beta$ is assumed constant here. A set of approximations are made to obtain the original predatorprey mode 11 . First, the ion and neutral fluxes at the boundaries of the transition layer are assumed in the form: $\left.\left(n_{i} v_{i}\right)\right|_{0}=0,\left.\left(n_{i} v_{i}\right)\right|_{L}=n v_{i}$ and $\left.\left(n_{a} v_{a}\right)\right|_{0}=n_{a} v_{a}$ and $\left.\left(n_{a} v_{a}\right)\right|_{L}=0$, where $v_{i}$ is the final ion velocity and $n(t)$ is plasma density at the exit of the transition layer, at $x=L, n(t) \equiv n_{i}(t, L)$, and $N(t)$ is neutral density at $x=0, N(t) \equiv n_{a}(t, 0)$. Thus, these boundary conditions imply full ionization, $n_{a}(L)=0$, zero flux of the ions from the left boundary, $\left.\left(n_{i} v_{i}\right)\right|_{0}=0$, and full acceleration the transition layer, $\left.\left(n_{i} v_{i}\right)\right|_{L}=n v_{i}$. Then, the boundary values for the plasma and neutral densities are used to approximate the averages over the transition layer: $\left\langle n_{a} n_{i}\right\rangle \simeq n N,\left\langle n_{a}\right\rangle \simeq N$, and $\left\langle n_{i}\right\rangle \simeq n$. These steps lead to the 0-D ODE system 11

$$
\begin{aligned}
& \frac{d N}{d t}-\frac{1}{L} N v_{a}=-\beta N n \\
& \frac{d n}{d t}+\frac{1}{L} n v_{i}=\beta N n
\end{aligned}
$$


These equations have the equilibrium solution with $n_{e q}=v_{a} / L \beta$ and $N_{e q}=v_{i} / L \beta$. Considering the perturbations $(\widetilde{n}(t), \widetilde{N}(t)) \sim \exp (-i \omega t)$ near the equilibrium, $n=n_{e q}+$ $\widetilde{n}(t), N=N_{e q}+\widetilde{N}(t)$ one obtains stable oscillations with

$$
\omega^{2}=\beta^{2} n_{e q} N_{e q}=v_{i} v_{a} / L^{2}
$$

It was later noted $\frac{12}{12}$ that oscillations (time dependence) in $N$ are inconsistent with the constant mass rate boundary condition at $x=0,\left.\left(n_{a} v_{a}\right)\right|_{0}=N v_{a}=\dot{M} / A m_{a}=$ const, which means that the $N$ should be constant for the constant neutral flow velocity; $A$ is the crosssection area. A modification of the 0-D model was suggested in Ref. 13 where the value of neutral density at the left (entrance) side was fixed constant, $\left.\left(n_{a} v_{a}\right)\right|_{0}=N_{0} v_{a}$, and the value at the exit was assumed time dependent, $\left.\left(n_{a} v_{a}\right)\right|_{L}=N(t) v_{a}$, so the neutral balance equation takes the form

$$
\frac{d N}{d t}+\frac{1}{L}\left(N-N_{0}\right) v_{a}=-\beta N n .
$$

The plasma balance equation was also modified with an additional term due to the sheath losses at the lateral walls

$$
\frac{d n}{d t}+\frac{1}{L} n v_{i}+\frac{1}{d} n c_{s}=\beta N n,
$$

where sheath losses are estimated based on the Bohm condition for the ion velocity, where $d$ is the radial channel width and $c_{s}=\sqrt{T_{e} / m_{i}}$. With these modifications, the equilibrium value of the neutral density in equations (8-9) is only corrected by the sheath losses,

$$
N_{e q}=\frac{1}{\beta}\left(\frac{v_{i}}{L}+\frac{c_{s}}{d}\right),
$$

while the equilibrium value of plasma density can be very different from that in equation (5) and takes the form

$$
n_{e q}=\frac{v_{a}}{\beta L}\left(\frac{N_{0}}{N_{e q}}-1\right) .
$$

Note that in this model, the value of the neutral density at $x=0$ should be sufficiently large $N_{0}>N_{e q}=\left(v_{i} / L+c_{s} / d\right) / \beta$. Considering perturbations near $N_{e q}$ and $n_{e q}$ one obtains damped oscillations ${ }^{13}$

$$
\omega=-\frac{i}{2} \frac{v_{a}}{L} \frac{N_{0}}{N_{e q}} \pm \sqrt{\beta^{2} n_{e q} N_{e q}-\frac{1}{4}\left(\frac{v_{a}}{L}\right)^{2}\left(\frac{N_{0}}{N_{e q}}\right)^{2}} .
$$

The essential problem of the predator-prey model is that it does not predict instability so no condition for the oscillations can be determined. It was argued that the electron 
dynamics should be important and more complex models were proposed such as using the electron (instead of ion) continuity equation with the drift-diffusion approximation for the electron velocity $\sqrt{19}$, , ion flows ${ }^{14}$, electron energy evolution ${ }^{13222}$, and two-zone mode ${ }^{16 \mid 17}$. Ref. 23 provides an overview of various models and involved mechanisms.

\section{CONTINUUM (1-D) PREDATOR-PREY MODEL}

Ideally, the reduction from the continuous 1-D model to the 0-D models as was discussed in the previous section should preserve the essential properties and features of the more complete model and do not introduce any fundamental changes to the 1-D model. Therefore, is of interest to study if the 1-D model has the properties predicted by the 0-D models. Here we discuss properties of the stationary and time-dependent solutions of the one-dimensional (continuous) model consisting of the continuity equations (1) and (2) for atoms and ions with constant flow velocities $v_{a}$ and $v_{i}$, and the ionization rate $\beta$. These are the same assumptions with which the original predator-prey model was derived. The stationary problem is formulated as two coupled equations:

$$
\begin{aligned}
& v_{a} \frac{\partial n_{a}}{\partial x}=-\beta n_{a} n_{i} \\
& v_{i} \frac{\partial n_{i}}{\partial x}=\beta n_{a} n_{i} .
\end{aligned}
$$

The exact solution to this system is found in the form:

$$
\begin{aligned}
& n_{a, s t}(\xi)=n_{a 0}\left(1+\frac{n_{0} v_{i}}{n_{a 0} v_{a}}\right) \frac{1}{\left(n_{0} v_{i}\right) /\left(n_{a 0} v_{a}\right) \exp (\xi)+1}, \\
& n_{i, s t}(\xi)=n_{0}\left(1+\frac{n_{a 0} v_{a}}{n_{0} v_{i}}\right) \frac{\exp (\xi)}{\exp (\xi)+\left(n_{a 0} v_{a}\right) /\left(n_{0} v_{i}\right)},
\end{aligned}
$$

where $n_{0}, n_{a 0}$ are values of ion density and atom density, respectively, at the left boundary, and normalized length is $\xi=x / l_{0}$, with $l_{0}=v_{i} v_{a} / \beta\left(n_{0} v_{i}+n_{a 0} v_{a}\right)$. It can be noted that the solutions of the stationary problem depend only on two parameters, $n_{0}, n_{a 0}$. Typical stationary solutions for $v_{i} / v_{a}=10$ are depicted in Figs. 1a $1 \mathrm{~b}$ by solid lines. Note that the position of the crossing point, where the ion and neutral densities are equal, and the localization of the ionization source $\beta n n_{a}$ depend on the value $n_{0}$ : they move to the right with decreasing $n_{0}$, and goes to infinity for $n_{0} \rightarrow 0$.

Numerical studies of the time-dependent equations (1) and (2) were performed with fixed boundary values at the left $n_{0}, n_{a 0}$ and free boundary conditions (spatial second derivative is 


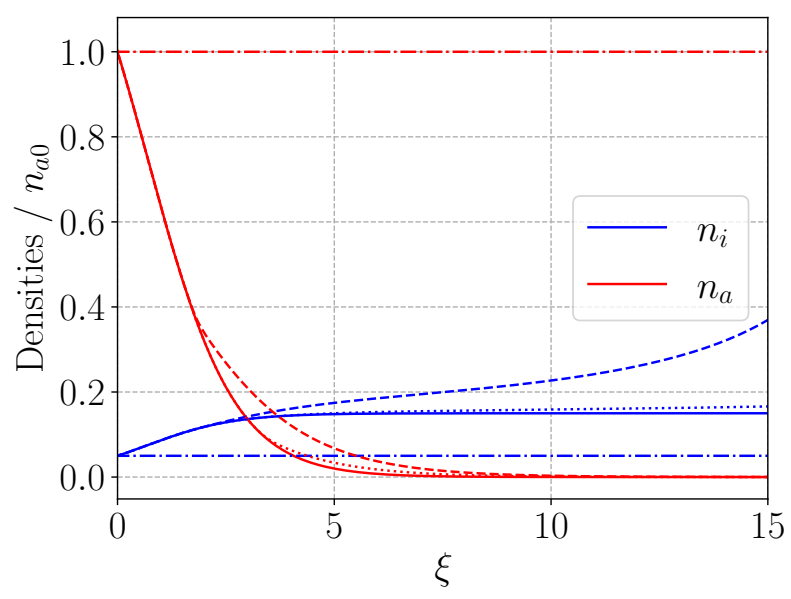

(a) $n_{0} / n_{a 0}=0.05, l_{0}=1.31 \mathrm{~cm}$

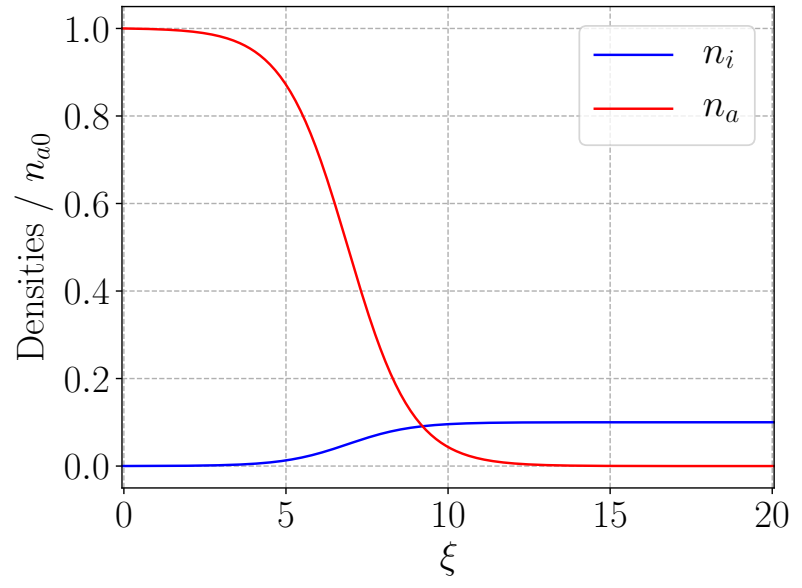

(b) $n_{0} / n_{a 0}=0.0001, l_{0}=1.96 \mathrm{~cm}$

Figure 1. Stationary solutions given by Eqs. (13) and (14) are shown by solid lines, with higher (a) and lower (b) ion density at the left boundary $n_{0}$. The time dependent solutions of Eqs. (1), and (2), converging to the stationary solution are shown for the case (a) at three consecutive time steps $t_{1}<t_{2}<t_{3}$, dot dashed (initial condition), dashed, and dotted line, respectively.

zero) on the right end. This analysis shows that all perturbations are damped and converge to the stationary solutions given by equations $(15)$ and $(16)$, as shown in Fig. 1a at different times. Thus, the 1-D predator-prey model with a constant spatial profile of the ion velocity does not exhibit oscillatory or unstable behaviour, unlike zero-dimensional predator-prey models. The 1-D continuum model presented here in some sense is similar to the asymptotic low-frequency model derived in the limit $n / N_{a} \ll 1 \underline{1612}$, which also predicts the neutral oscillations with the frequencies of the order of given by equation (7). Contrary to ${ }^{6 / 12}$, our model does not show neutrally stable oscillations but only damped modes.

One can envisage that the boundary value of plasma density could be perturbed, e.g. by some sheath instability. Such perturbations may also arise in the experiments with segmented anode ${ }^{24}$, external modulations of the anode voltage ${ }^{25}$, and two-stage thruster configurations 26 . Therefore, it is interesting to investigate how this nonlinear system responds to external perturbations of the ion density and whether such external perturbations may grow in amplitude while propagating from the left boundary. We will impose harmonic external modulations of the ion density at the left boundary in the form $n_{i}(0)=n_{i 0}\left(1+r \sin 2 \pi f_{\text {ext }} t\right)$, where $f_{\text {ext }}$ is the modulation frequency, with $r=0.15$. We 
consider the 1-D continuum model, Eqs. (1]2) with $v_{i}=10 v_{a}, n_{i 0} / n_{a 0}=0.05, l_{0}=1.31 \mathrm{~cm}$, Fig. 1a, with the simulation length $15 l_{0}$. Since velocities of neutrals and ions are different, there are two natural modes that can be excited during the external modulations, with the wavelengths given with $\lambda_{a}=v_{a} / f_{\text {ext }}$ and $\lambda_{i}=v_{i} / f_{\text {ext }}$. We consider four values of the driving frequencies $f_{\text {ext }} l_{0} / v_{a}=(0.1,0.5,1,10)$. For these values, the corresponding natural wavelengths of the neutral and ion characteristic wavelengths are $\lambda_{a}=(10,2,1,0.1) l_{0}$ and $\lambda_{i}=(100,20,10,1) l_{0}$, respectively.

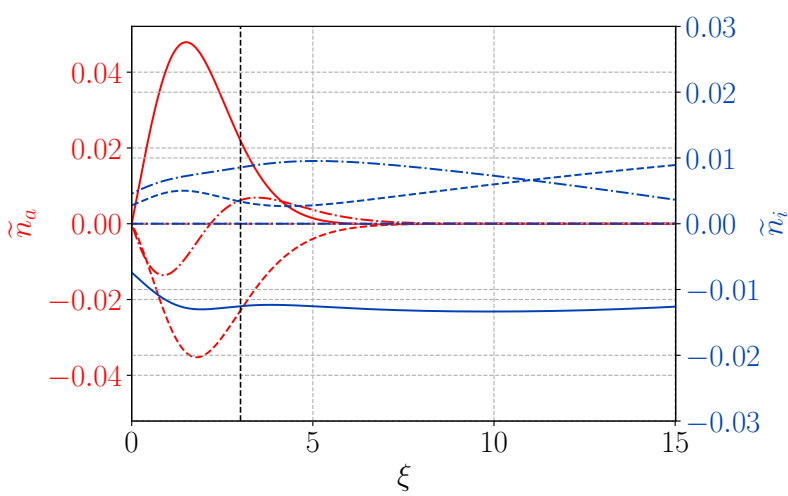

(a) $f_{\text {ext }} l_{0} / v_{a}=0.1$

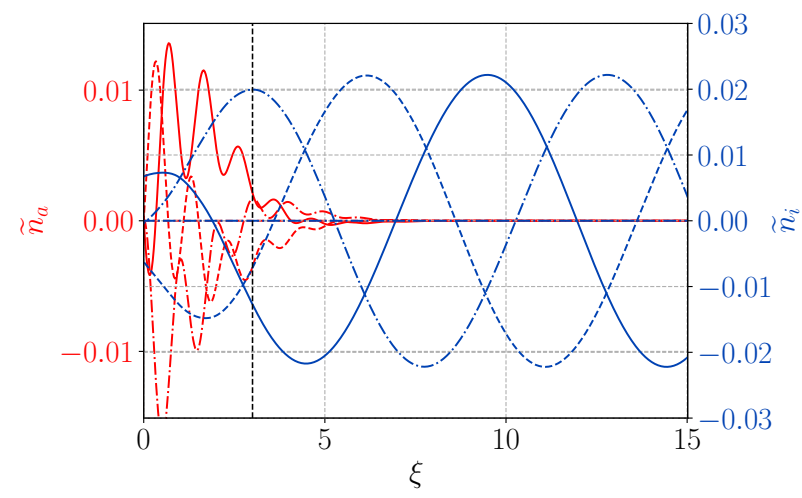

(c) $f_{\text {ext }} l_{0} / v_{a}=1$

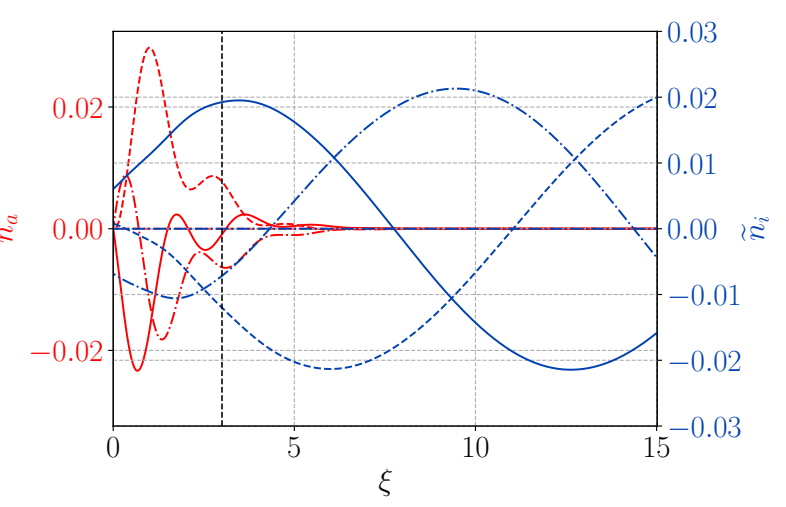

(b) $f_{\text {ext }} l_{0} / v_{a}=0.5$

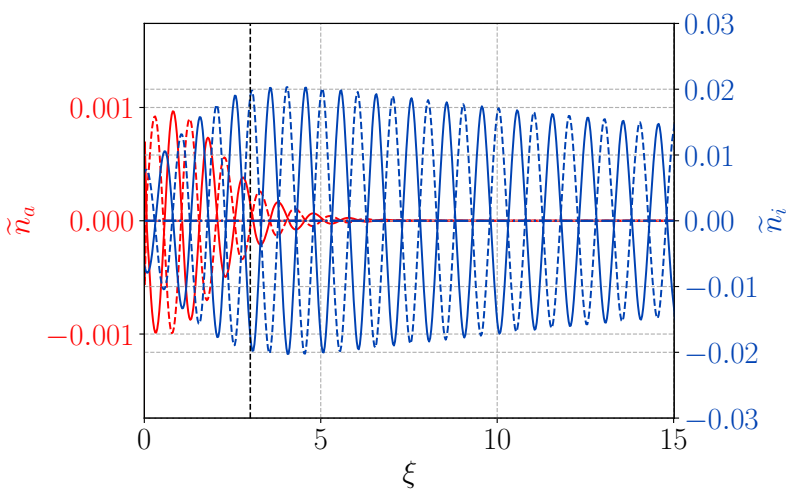

(d) $f_{\text {ext }} l_{0} / v_{a}=10$

Figure 2. Instantaneous profiles of perturbed ion $\widetilde{n}_{i}$ (blue) and neutral $\widetilde{n}_{a}$ (red) densities for the various driving frequencies $f_{\text {ext }} l_{0} / v_{a}=(0.1,0.5,1,10)$, shown, respectively, in (a), (b), (c), (d). Three consecutive time snapshots $t_{1}<t_{2}<t_{3}$ are shown for each variable, depicted with the dot dashed line, dashed line, and solid line, respectively; they divide one oscillation period in 3 equal intervals. Crossing point (of stationary ion and atom densities profiles) location is shown with the black dashed line. 
The resulting response of the system of Eqs. (1/2) under the external modulations of the ion density is presented in Fig. 2 for the perturbed variables, defined as $\widetilde{n}=n-n_{s t}$, where $n_{s t}$ is the corresponding stationary solution. It can be noted that in all cases ion density perturbations grow in absolute values before reaching the crossing point and leaving the ionization region, whereas the amplitude of neutral density perturbations decays, which is expected in the ionization region. Note that while the absolute value of the perturbations of the ion density seems increasing, the relative value $\widetilde{n} / n_{s t}$ does not grow. Thus, in the presence of external ion density perturbations in the near-anode region, the system exhibit externally driven oscillations that are advected along the channel but show a limited increase of the amplitude. However, the atom response is slightly more complicated and besides the natural mode can exhibit the nonlinear response (on ion natural wavelength). It is also affected by the atom stationary profile (Fig. 1a), as atom perturbations decay rapidly after the crossing point. It is seen that for the low frequency perturbations the natural atom mode is excited (Fig. 2a), while for the higher frequencies the ion characteristic wavelengths become more and more dominant in the atom response (seen gradually through Figs. 2b2d). We remind that the amplitude of the ion density perturbations was fixed in these cases. The amplitude of the neutral density perturbations is maximal for the lower frequencies. We have also investigated the case when the neutral density was modulated at the left boundary. Such a system shows similar behaviour and does not show the unstable modes.

\section{IONIZATION OSCILLATIONS IN THE 1-D CONTINUOUS MODEL WITH THE ION BACK-FLOW IN THE NEAR ANODE REGION}

As it was discussed in the previous section, the reduced fluid model, described with Eqs. (1, 2), with spatially uniform profiles of $v_{i}, v_{a}$, and $\beta$ is stable. Here we will show that the same system with spatial dependence of $v_{i}$, when $v_{i}$ is negative near the anode, the so-called ion back-flow, becomes unstable and exhibit self-consistent oscillations. The ion

back-flow region naturally forms in the presheath, a quasineutral region near the anode with the negative electric field due to the electron diffusion 27 30 . The positive density gradient near the anode creates a strong electron flow to the anode. To maintain ambipolarity (the total current remains uniform), the ion back-flow current occurs to compensate the increase of the electron current. The current ambipolarity is required to keep plasma quasineutral. 
This process is somewhat similar to the formation of the electric field in the presheath region near the plasma boundary where the electric field is induced to accelerate ions toward the boundary (up to the Bohm velocity) to balance electron and ion losses.

In this configuration the only fixed boundary condition is a value of the neutral density on the left, as their characteristics travel strictly to the right (atom velocity is a positive constant). Ion density boundary condition is free on the left, $\partial_{x}^{2} n_{i}(0)=0$. First, we consider the simple configuration with the velocity profile $v_{i}$ as a strictly linear function of the position. The ionization rate $\beta$ is taken uniform. One might argue the constant $\beta$ is an unrealistic assumption for a typical Hall thruster where electron energy near the anode is low due to higher mobility, shaping the $\beta$ profile; we consider these effects in Section $\mathrm{V}$ in the comparison with the full fluid model. Recombination of plasma at the anode is not included here: the ion flux converted to the neutral flux at the anode is not added to the neutral flow. It can also be included, increasing the amplitude of oscillations, but it is not required for the existence of the oscillations. An example of such oscillations shown for a typical Hall thruster parameters (the stationary plasma thruster "SPT 100" channel length $3 \mathrm{~cm}$, ionization rate $\beta=9 \times 10^{-14} \mathrm{~m}^{3} \mathrm{~s}^{-1}, v_{a}=150 \mathrm{~m} / \mathrm{s}$. Atom density at the anode $n_{a 0}$ is evaluated from $n_{a 0} v_{a}=\dot{m} / A m_{a}$, assuming $\dot{m}=5 \mathrm{mgs}^{-1}$, Xenon atom mass, and $A=12.75 \pi \mathrm{cm}^{2}$. For the simplicity, we assumed the linear ion flow velocity profile $v_{i}=(2.5 x-1.5) \mathrm{km} / \mathrm{s}(x$ is in $[\mathrm{cm}])$, with the ion back-flow extent $L_{b}=0.6 \mathrm{~cm}$. The resulting time and space evolution of ion and atom densities is shown in Fig. 3 , The observed frequency is $12.8 \mathrm{kHz}$, in the range of the values observed in experiments and simulations for breathing mode.

Now, for the same setup with ion velocity profile in the form $v_{i}=(2.5 x-1.5) \mathrm{km} / \mathrm{s}(x$ is in $[\mathrm{cm}]$ ), we will show effects of the amplitude of the ionization rate $\beta$, which is still assumed uniform here for simplicity. The ionization rate coefficient $\beta$ needs to be sufficiently large to support plasma discharge. Interestingly, in addition to a threshold $\beta$ value for the discharge to exist, there is also a threshold between stable and oscillatory plasma behaviour. In the oscillatory regime, there is a value of $\beta$ when the amplitude of the oscillations is the largest (note that here we assume that $\beta$ is constant along the channel). It shouldn't be of surprise, as when the values of $\beta$ are too high, the ionization takes place immediately near the left end and so both density profiles have maximum values at the left end. The dependency of the oscillation amplitude on $\beta$ is shown in Fig. 4a, where the peak-to-peak amplitude of the 


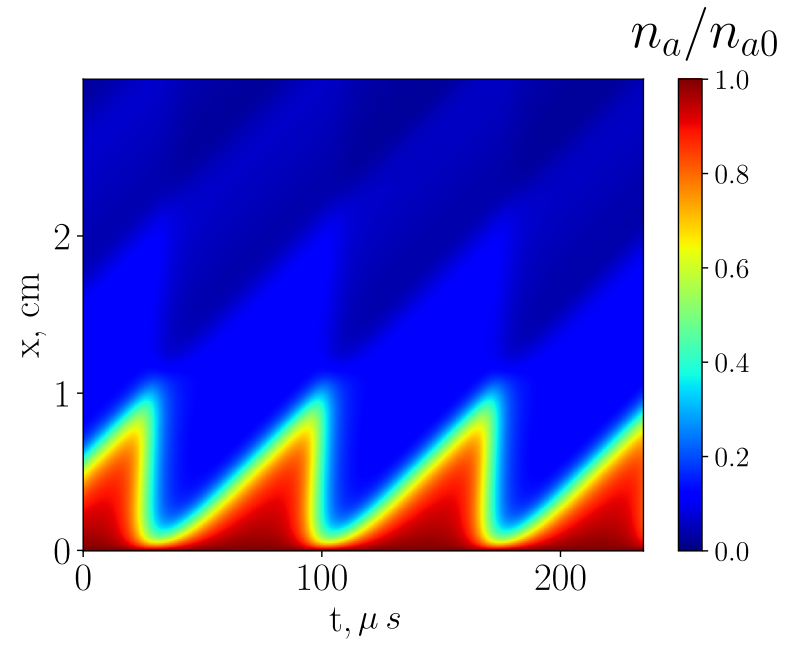

(a)

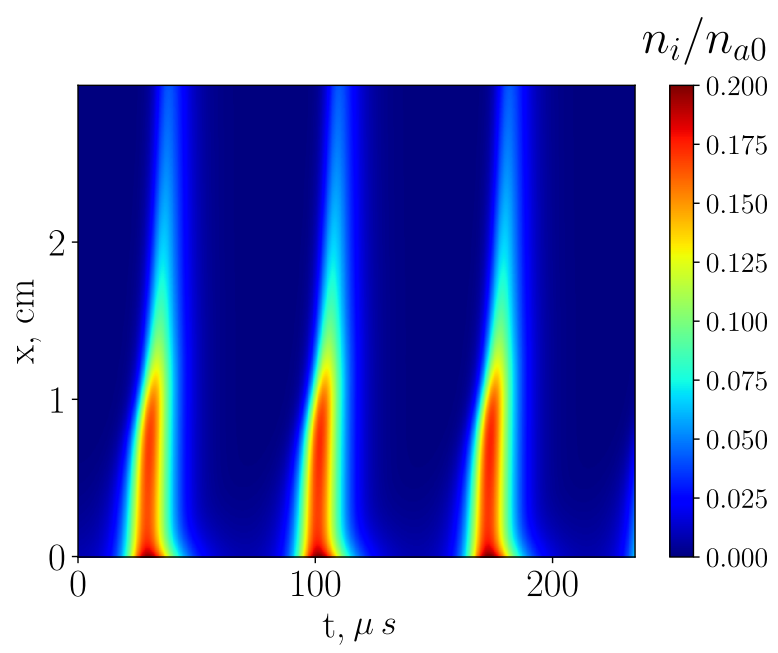

(b)

Figure 3. Evolution in space and time of atom (a) and ion (b) density, normalized to $n_{a 0}$.

averaged in space density $\left\langle n_{i}\right\rangle_{x}(t)$ is shown.

The frequency dependence on $\beta$ is presented in Fig. $4 \mathrm{~b}$. If the ion back-flow region is very short, i.e. $v_{i}(0) \approx 0$, the ionization mostly will occur at the anode for the same reasons, and there would not be enough spatial separation between high and low atom density points. Dependencies, similar to those shown in Figs. 4a, 4b, are also observed for different values of the ion back-low extent $L_{b}$. They follow the same scaling as presented below in Figs. $5 \mathrm{~b}$, 6.

The effect of the ion back-flow region is investigated further for our reduced model by taking the ion velocity profiles with a variable ion back-flow extent. The Fig. 5a shows the velocity profiles taken as parabolas specified with the three points, $v_{i}(0)=-1.5 \mathrm{~km} / \mathrm{s}$, $v_{i}(L)=20 \mathrm{~km} / \mathrm{s}$, and $v_{i}\left(L_{b}\right)=0 \mathrm{~km} / \mathrm{s}$, where $L_{b}$ is the length of the ion back-flow region, the distance from the anode to the transition point where the ion velocity reverse its sign. The value of $L_{b}$ was varied, with the system length set to $3 \mathrm{~cm}$, the ionization rate $\beta=$ $5 \times 10^{-14} \mathrm{~m}^{3} / \mathrm{s}$, atom flow velocity $v_{a}=150 \mathrm{~m} / \mathrm{s}$. Simulations using these velocity profiles reveal that that oscillation frequency scales with the atom fly-by frequency $v_{a} / L_{b}$ in the back-flow region, Fig. 5b. The oscillations amplitude decreases with decreasing $L_{b}$ and the oscillations disappear for $L_{b} \rightarrow 0$, as shown in Fig. 6. Also, no oscillations were observed for monotonically increasing ion velocity profiles with $v_{i}(0) \geq 0$.

Zero-dimensional predator-prey models predict that the frequency depends on the exhaust 


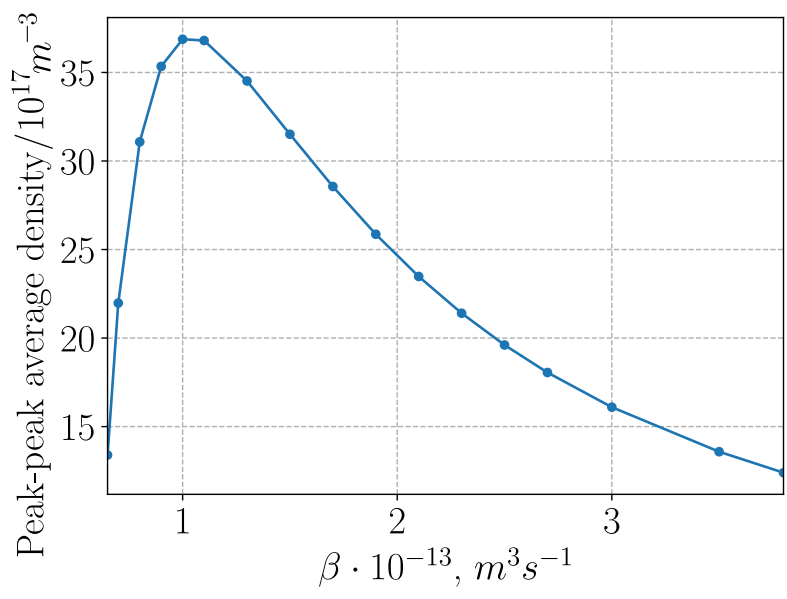

(a)

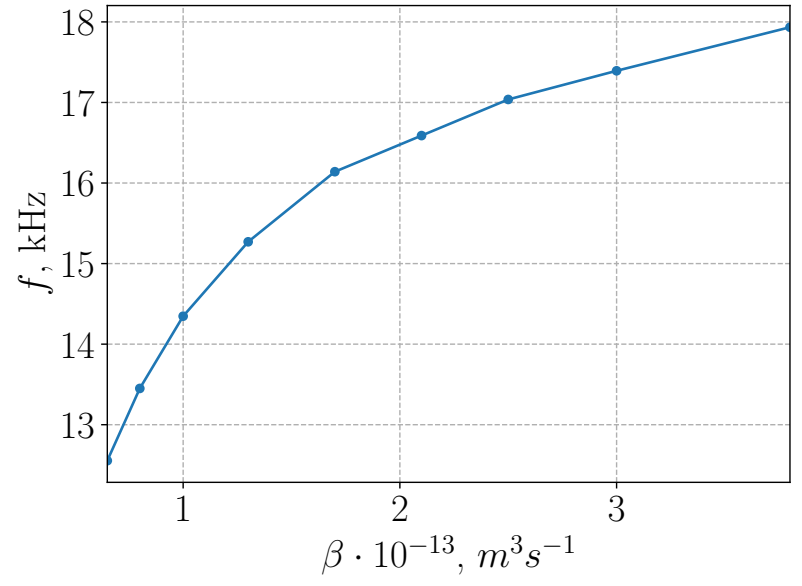

(b)

Figure 4. Peak-to-peak values of averaged in space ion density $\left\langle n_{i}\right\rangle_{x}(t)$ as a function of ionization rate (a). The main frequency component of observed oscillations as a function of the ionization rate $(b)$.

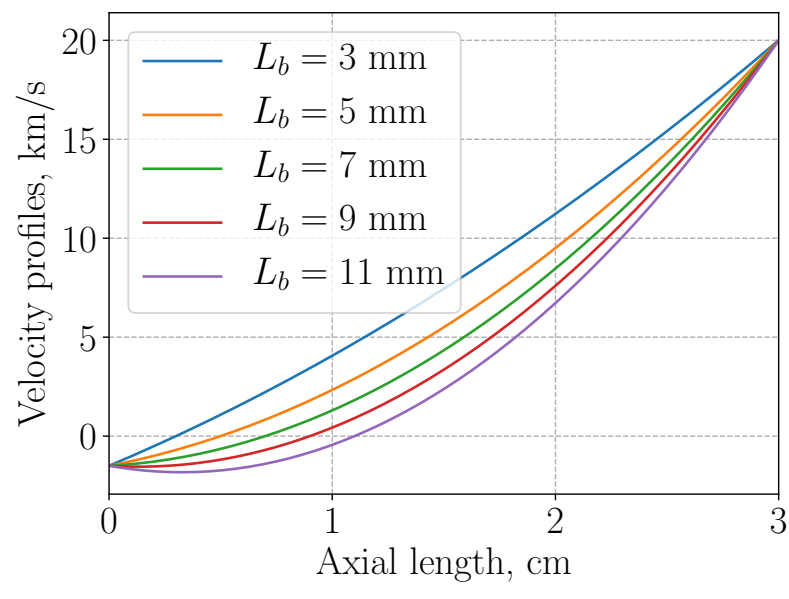

(a)

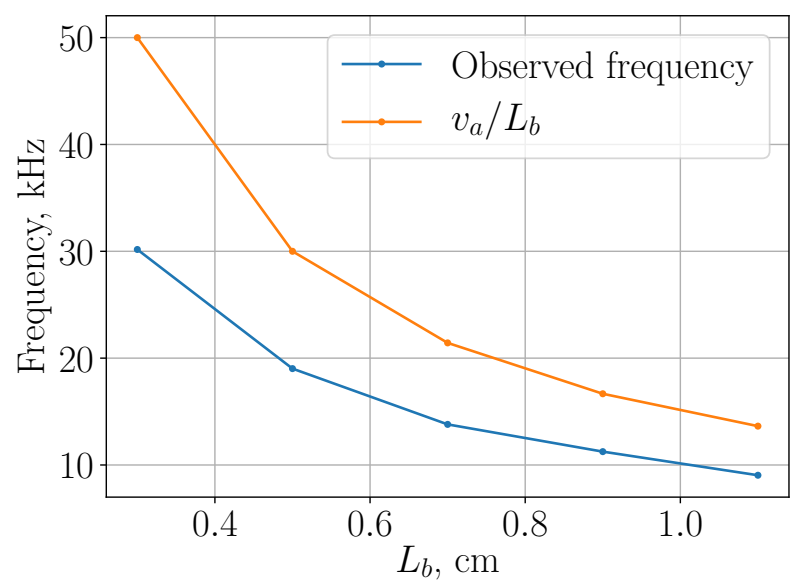

(b)

Figure 5. Velocity profiles (a) for a variable ion back-flow region $L_{b}$ while the exhaust velocity is fixed to $20 \mathrm{~km} / \mathrm{s}$ (300 eV is a typical ion energy in the exhaust plume of "SPT100" (4). The corresponding frequency (b) as a function of $L_{b}$ in compare with neutral flyby frequency of $v_{a} / L_{b}$ in the backflow region.

ion velocity. Here, we show that for our model this effect is very small and, mostly, the backflow region "defines" the frequency. Fig. 7a shows the family of the ion velocity profiles, constructed in a similar manner (by a parabola) but keeping the back-flow length the same 


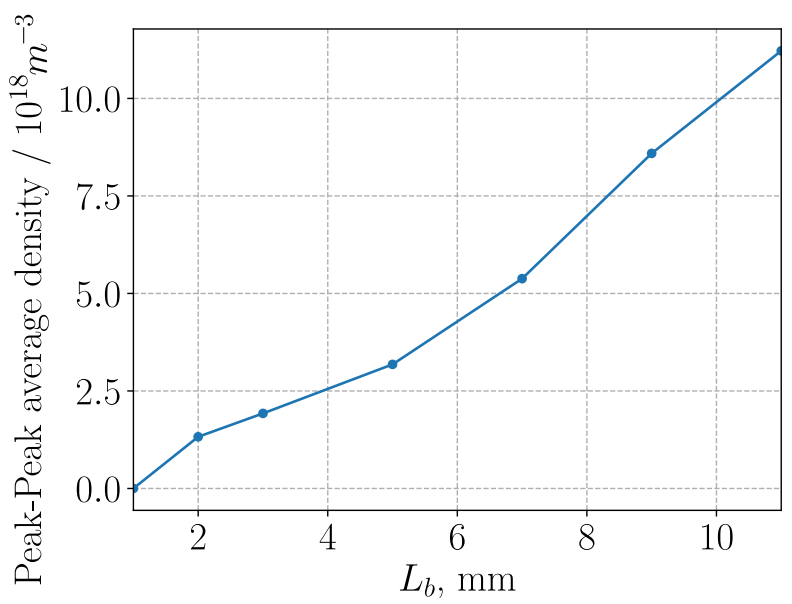

Figure 6. Peak-to-peak values of averaged in space ion density $\left\langle n_{i}\right\rangle_{x}(t)$ as a function of the ion back-flow region $L_{b}$. Oscillations disappear as $L_{b}=1 \mathrm{~mm}$ goes to zero.

$(1 \mathrm{~cm})$ and varying $v_{i}(L)=(14,18,22,26) \mathrm{km} / \mathrm{s}$. The resulting frequency for all these profiles was found to be close to $10 \mathrm{kHz}$, Fig. $7 \mathrm{~b}$, predicted by the previous example in Fig. $5 \mathrm{~b}$ with the $L_{b}=1 \mathrm{~cm}$.

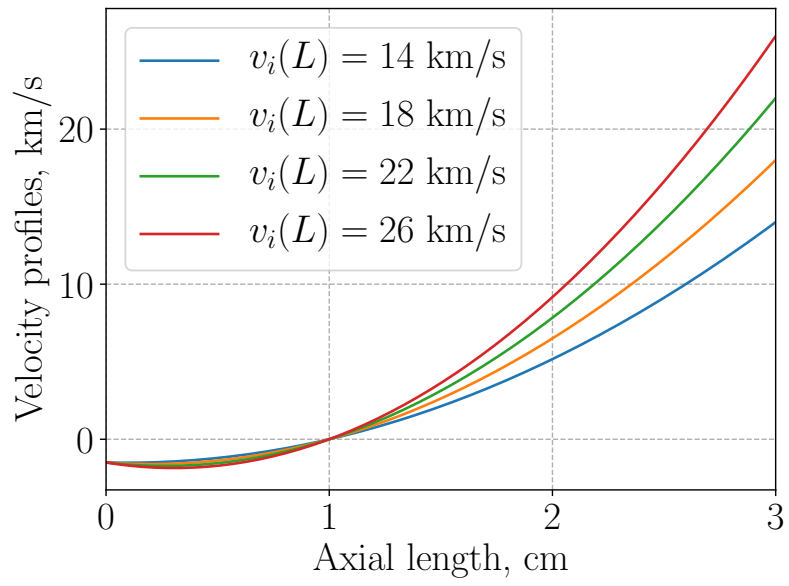

(a)

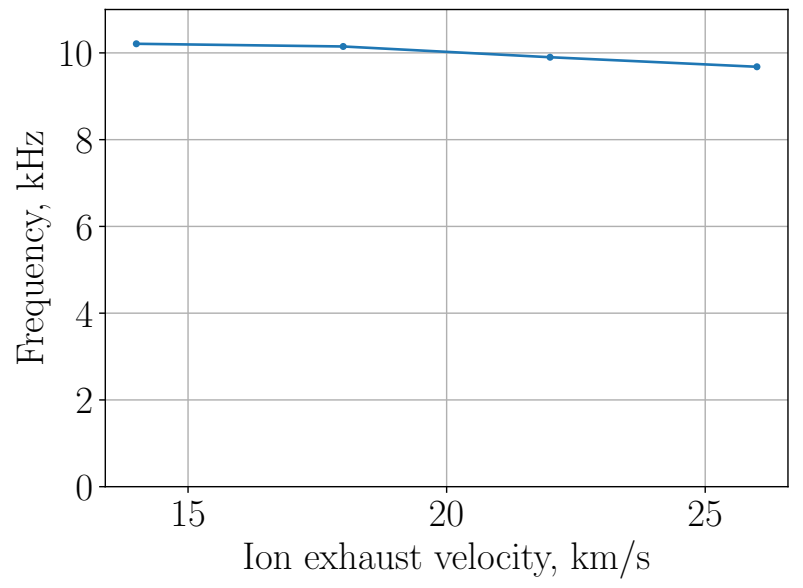

(b)

Figure 7. Velocity profiles (a) and corresponding frequency (b) as a function of $v_{i}(L)$, for the variable ion exhaust velocity $v_{i}(L)$ while keeping the back-flow region $L_{b}$ constant.

The mechanism of the oscillations can be considered in three main stages, starting from a stationary state in Fig. 1a, but with the ion velocity back-flow region. In the first stage we observe enhanced ionization process in the region of plasma stagnation $\left(v_{i} \approx 0\right)$. The 
ionization process in this stage may be approximated as $\dot{n}_{i}=\beta n_{i} n_{a}, \dot{n}_{a}=-\beta n_{i} n_{a}$ (where $n_{i}, n_{a}$ are some average plasma and atom densities, respectively, in that region). In this stage, effects of atom inflow and ion outflow are relatively small here. The ionization proceeds with the exponential rise plasma density $n_{i} \sim \exp \left(\beta n_{a} t\right)$ and depletion of atom density. In the second stage, the newly created plasma peak splits and partially moves to the anode (via back-flow) wiping down the rest of atoms near the anode. Finally, in the third stage, the system enters the period of "regeneration" with both plasma and atom densities low, while atoms slowly fill the region near the anode. The atom density remains higher than the ion density, and the ion density balance is predominantly follows the equation $\dot{n}_{i}=$ $\beta n_{i} n_{a}-\gamma_{l o s s} n_{i}$, where $\gamma_{l o s s} n_{i}$ represents the ion advection losses via the back-flow. In this quasistationary state, there is no exponential rise of ionization but slow inflow of atoms (to the right) filling this region toward the stagnation region. The third stage is also the slowest and defines the breathing mode period, hence the backflow lengths defines the period (Fig. 5b). Additionally, if the recombination at the anode is included, it increases a number of atoms injected to the system for the stage three, and it increases the amplitude of the oscillations.

\section{REDUCED VS FULL SELF-CONSISTENT MODEL}

In this section we compare the reduced and full models for the breathing modes, and demonstrate that our simple reduced model, Eqs. (1 2), reproduces well the results from the

full self-consistent time-dependent mode1 ${ }^{18}$. First, we give a brief description of the full fluid self-consistent model for low-frequency ionization oscillations.

\section{A. Full self-consistent model}

The full model of low-frequency axial plasma dynamics in Hall thruster is considered in electrostatic and quasineutral approximation for three species: neutrals, ions, and electrons. Our model is fully fluid both for the ion, neutral, and electron components. The electron equations in general are similar to those used in Refs. $\underline{6] 7}$. The full fluid model consists of time-dependent PDE equations for neutral atom density $n_{a}$, plasma density $n$ (ion and 
electron), ion flow velocity $v_{i}$, and electron temperature $T_{e}$ :

$$
\begin{aligned}
& \frac{\partial n_{a}}{\partial t}+v_{a} \frac{\partial n_{a}}{\partial x}=-\beta n_{a} n \\
& \frac{\partial n}{\partial t}+\frac{\partial}{\partial x}\left(n v_{i}\right)=\beta n_{a} n \\
& \frac{\partial v_{i}}{\partial t}+v_{i} \frac{\partial v_{i}}{\partial x}=\frac{e}{m_{i}} E+\beta n_{a}\left(v_{a}-v_{i}\right), \\
& \frac{3}{2} \frac{\partial}{\partial t}\left(n T_{e}\right)+\frac{5}{2} \frac{\partial}{\partial x}\left(n v_{e x} T_{e}\right)+\frac{\partial q_{e}}{\partial x}=n v_{e x} E-n n_{a} \mathrm{~K}-n \mathrm{~W}
\end{aligned}
$$

where the electric field $E$ is obtained from the electron momentum balance equation

$$
v_{e x}=-\mu_{e} E-\frac{\mu_{e}}{n_{e}} \frac{\partial\left(n T_{e}\right)}{\partial x}
$$

obtained with neglected electron inertia, where $\mu_{e}$ is the electron mobility across the magnetic field (described below). Quasineutrality condition, along with the constraint $\int_{L} E d x=$ $U_{0}\left(U_{0}\right.$ is the applied potential) leads to the total current $J_{T}=e n\left(v_{i}-v_{e}\right)$

$$
J_{T}=\frac{U_{0}+\int_{0}^{L}\left(\frac{v_{i}}{\mu_{e}}+\frac{1}{n} \frac{\partial p_{e}}{\partial x}\right) d x}{\int_{0}^{L} \frac{d x}{e n \mu_{e}}}
$$

which is spatially uniform but may oscillate in time. Other quantities in the system 17 . 20 are the constant atom flow velocity $v_{a}$, the ionization rate coefficient $\beta$ (obtained with BOLSIG $+\sqrt{31}$ for Maxwellian EEDF using SIGLO database ${ }^{(32}$ ), the elementary charge $e$, the electron mass $m_{e}$, the ion (Xenon) mass $m_{i}=131.293 \mathrm{amu}$, the anomalous energy loss coefficient ${ }^{7} \mathrm{~W}$, the collisional energy loss coefficient $\mathrm{K}$ (also via BOLSIG+), and the electron heat flux given by

$$
q_{e}=-\frac{5}{2} \mu_{e} n T_{e} \frac{\partial T_{e}}{\partial x}
$$

The electron transport across magnetic field is described in the form of the magnetized mobility

$$
\mu_{e}=\frac{e}{m_{e} \nu_{m}} \frac{1}{1+\omega_{c e}^{2} / \nu_{m}^{2}},
$$

where $\nu_{m}$ the total electron momentum exchange collision frequency, $\omega_{c e}=e B / m_{e}$ is the electron cyclotron frequency. In this model $\nu_{m}$ is represented in the form as was adopted in Ref! 33 ,

$$
\nu_{m}=\nu_{e n}+\nu_{\text {walls }}+\nu_{B},
$$


where the electron-neutral collision frequency $\nu_{e n}$, electron-wall collision frequency $\nu_{\text {walls }}$, and anomalous Bohm frequency $\nu_{B}$ are given with:

$$
\begin{array}{r}
\nu_{e n}=k_{m} n_{a}, \\
\nu_{\text {walls }}=\alpha 10^{7}\left[\mathrm{~s}^{-1}\right], \\
\nu_{B}=\left(\beta_{a} / 16\right) e B / m_{e} .
\end{array}
$$

where $k_{m}=2.5 \times 10^{13} \mathrm{~m}^{-3} \mathrm{~s}^{-1}, \alpha$ and $\beta_{a}$ are adjusting constants. The profile of external magnetic field $B$ is shown if Fig. 8a, with the channel's exit in the peak of magnetic field intensity, given by $B=B_{0} \exp \left(-(x-l)^{2} / 2 \delta_{B}^{2}\right)$, where $l=2.5 \mathrm{~cm}$ is the channel length and $\delta_{B}$ defines a magnetic field profile width. This model use different parameters inside $(x<l)$ and outside $(x \geq l)$ the channe $\sqrt{33 \sqrt[35]{3}}$, the near wall conductivity contribution (27) $\alpha_{\text {in }}=0.2, \alpha_{\text {out }}=0$, and the anomalous contribution (28) is set to $\beta_{a, \text { in }}=0.1, \beta_{a, \text { out }}=1$. The anomalous electron energy loss coefficient ${ }^{77} \mathrm{~W}$ is modeled as

$$
\mathrm{W}=\nu_{\varepsilon} \varepsilon \exp (-U / \varepsilon),
$$

where $\varepsilon=3 / 2 T_{e}, U=20 \mathrm{~V}$, and $\nu_{\varepsilon}$ is electron energy anomalous loss coefficient. A constant mass flow rate $\dot{m}$ determines the value of $n_{a}$ at the boundary together with the recombination of plasma that flows to the anode, hence the boundary condition:

$$
n_{a}(0)=\frac{\dot{m}}{m_{i} A v_{a}}-\frac{n v_{i}(0)}{v_{a}},
$$

where $A$ is the anode surface area of a thruster. Bohm type condition for ion velocity can be imposed at the anode $v_{i}(0)=-b_{v} \sqrt{T_{e} / m_{i}}$, where $b_{v}=0-1$ is the Bohm velocity factor which can be varied. Both anode and cathode electron temperature are fixed with $T_{e}(0)=T_{e}(L)=2 \mathrm{eV}$. All other boundary conditions are free (spatial second derivative is zero).

Following the LANDMARK benchmark ${ }^{36}$ Test Case 3, we use the parameters that result in bulk low-frequency oscillations, with the electron energy anomalous loss coefficient inside the channel $\nu_{\varepsilon, \text { in }}=10^{7} \mathrm{~s}^{-1}$ and outside $\nu_{\varepsilon, \text { in }}=0.4 \cdot 10^{7} \mathrm{~s}^{-1}$. The resulting plasma currents evolution shown in Fig. 8b, assuming the typical inner and outer radius of a Hall thruster ("SPT 100" ${ }^{4}$ ) $R_{1}=3.5 \mathrm{~cm}, R_{2}=5 \mathrm{~cm}$, respectively. The input atom mass flow used in the simulation is $\dot{m}=5 \mathrm{mgs}^{-1}$ corresponds to $I=\dot{m} q_{e} / m_{i}=3.67 \mathrm{~A}$. The average ion current (to the exit plane) is $3.68 \mathrm{~A}$ which is consistent with the mass flow rate. The electron current 
(to the exit plane) is of the same order (or slightly larger). Other simulation parameters for this test case are: $\delta_{B, \text { in }}=1.1 \mathrm{~cm}, \delta_{B, \text { out }}=1.8 \mathrm{~cm}, A=\pi\left(R_{2}^{2}-R_{1}^{2}\right), b_{v}=1$.

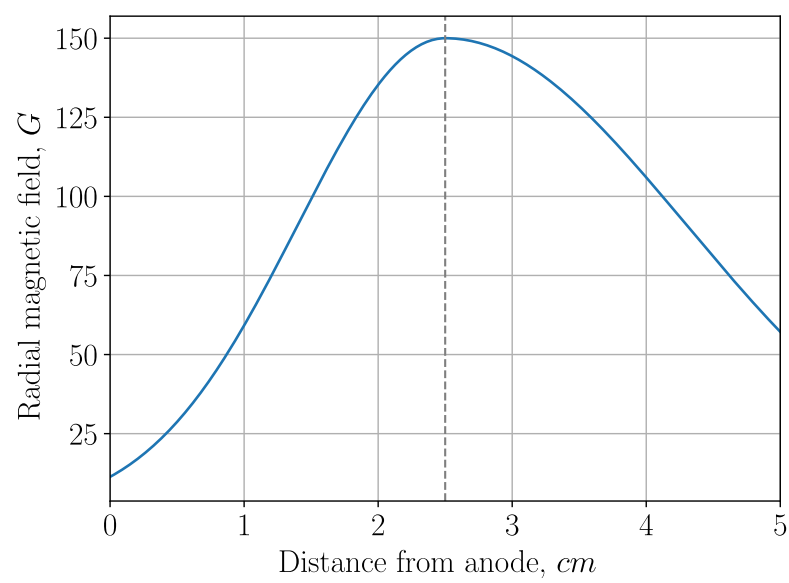

(a)

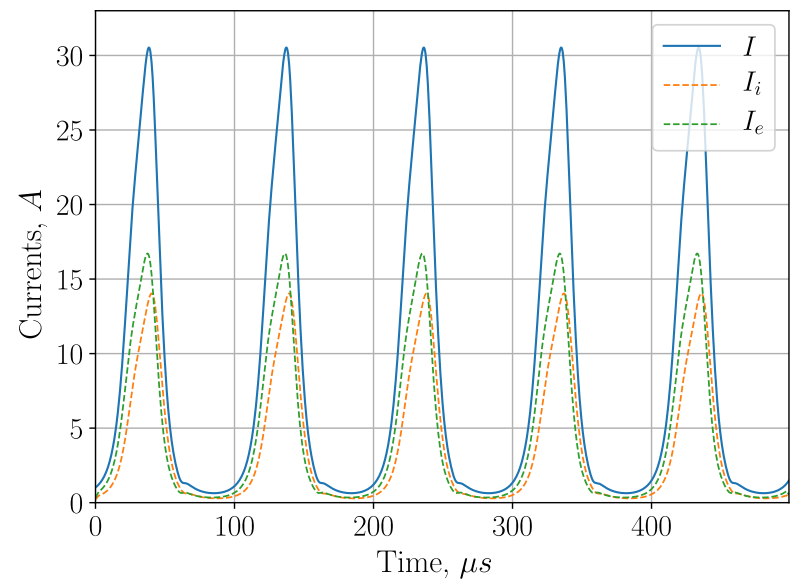

(b)

Figure 8. The magnetic field profile (a) used in full fluid and hybrid simulations, with the channel exit located $2.5 \mathrm{~cm}$ from anode (dashed line). Low-frequency oscillations in time (b) of total current $I$, ion current $I_{i}$ (at $x=5 \mathrm{~cm}$ ), and electron current $I_{e}$ (at $x=5 \mathrm{~cm}$ ) in the full fluid model using specified parameters.

The self-consistent fluid model used here is essentially based on the formulations and parameters suggested in Refs. $\frac{5 / 733 / 35}{\text {, also see the LANDMARK benchmark }}$. The fluid model has been compared against the hybrid model ${ }^{33137}$; both models demonstrate similar results 18 .

\section{B. Comparison of the reduced model with predictions of the full self-consistent model}

We compare now results of the full fluid model (in the regime described above), with the reduced model. In the full model the ion velocity profile $v_{i}$ and the ionization rate $\beta$ profile are self-consistent variables, and for the reduced model we take averaged in time profiles of these variables, Figs. 9a, 9b. We also include the ion back-flow recombination to the atom boundary condition at the anode in the reduced model, described by Eq. (30), in agreement with the full model.

Time evolution of $n_{a}, n_{i}$, and the source $S=\beta n_{a} n_{i}$, are shown in Figs. 10a, 10d, 10g, 


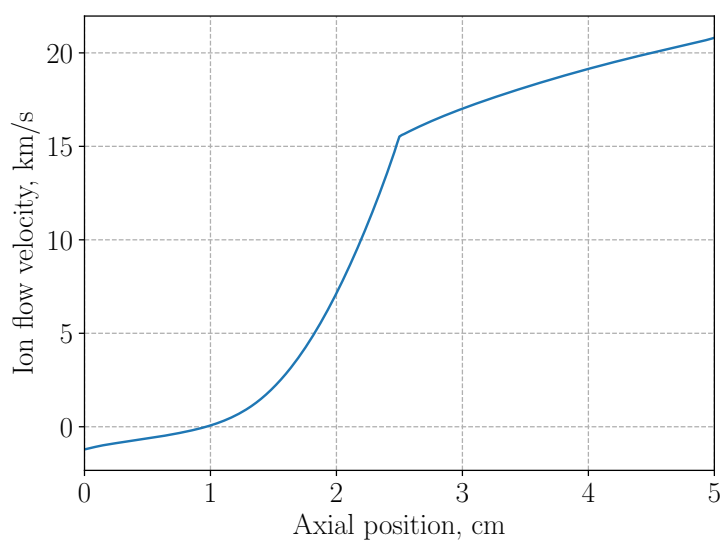

(a)

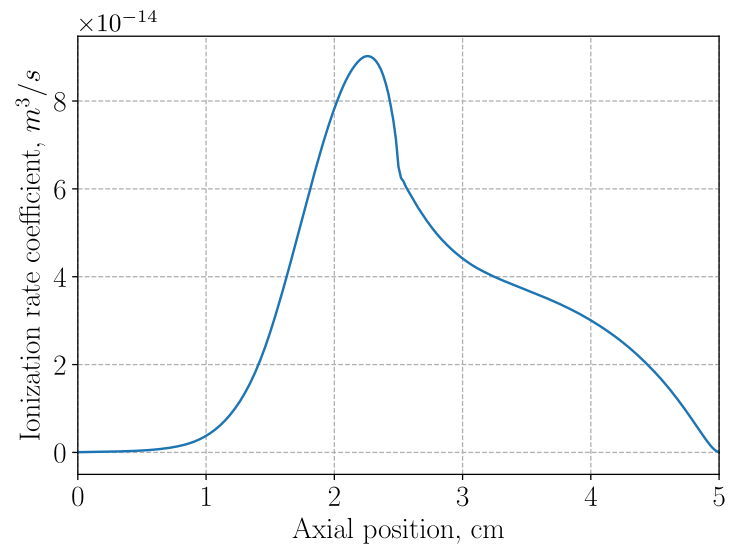

(b)

Figure 9. Ion velocity (a) and ionization rate (b) coefficient profiles obtained from the full fluid model as time-averaged over a few oscillation periods. They are used in the reduced model as fixed profiles in time.

respectively. The same quantities from the reduced model are shown in Figs. 10b, 10e, 10h, It is seen that the reduced model results in oscillatory behaviour, qualitatively reproducing the breathing mode oscillations, but with much higher amplitudes (about one order) of evolving variables. The observed natural frequency in this model is also higher, $14.9 \mathrm{kHz}$, compared to $10.2 \mathrm{kHz}$ in the full model.

The observed discrepancy can be explained by the absence of the electron temperature evolution and lack of self-consistent ionization rate dynamics in the reduced model which is known to affect the breathing mode characteristics, e.g. Ref. 38. One observation is that the average position of the source term $S$ in our reduced model is closer to the anode (Fig. 10h) (compared with the full model) that results in higher frequency. Higher amplitude may also be explained by the shift of the ionization source toward the region with higher ion back-flow velocity so that more ions return to the anode and recombine enhancing the positive feedback loop. We found that simply lowering the values of the ionization rate, precisely by taking $0.82 \beta$ of the original averaged $\beta$ profile (shown in Fig. 9b) agrees with the full model much closer, see Figs. 10c, 10f, 10i, with the oscillation frequency of $11.8 \mathrm{kHz}$. While our intention is not really to achieve full quantitative agreement, this exercise shows the sensitivity to the temperature effects. It is also possible that the strong nonlinear dependence of the ionization rate on temperature may suggest some weighting average for the ionization rate. We leave 
the investigation of the proper averaging technique for future work.

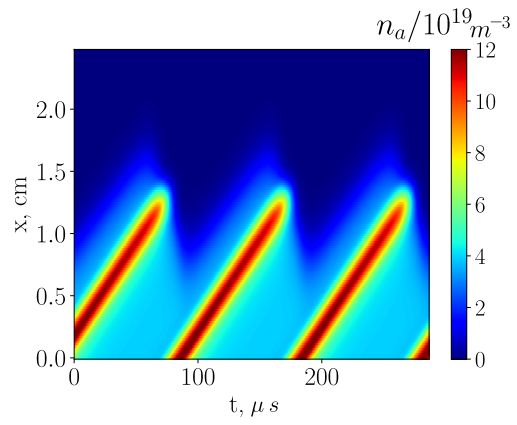

(a) $n_{a}$ (Full)

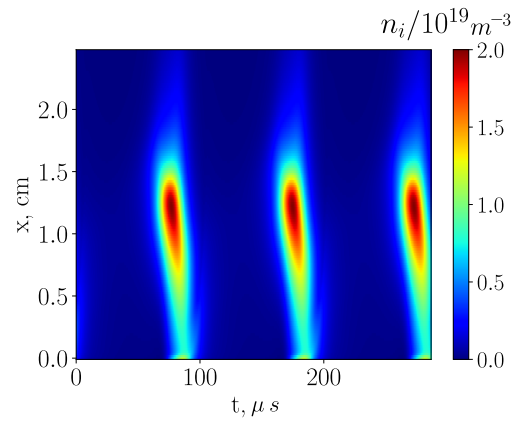

(d) $n_{i}$ (Full)

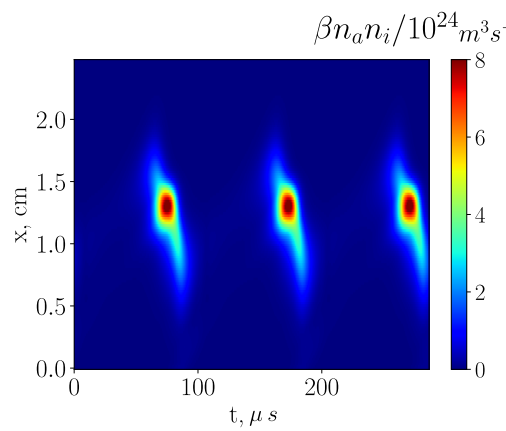

(g) Source (Full)

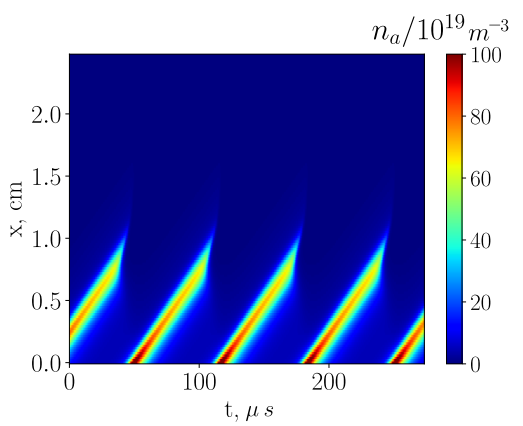

(b) $n_{a}$ (Reduced)

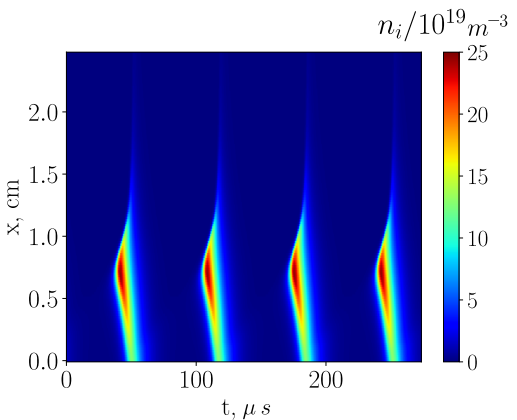

(e) $n_{i}$ (Reduced)

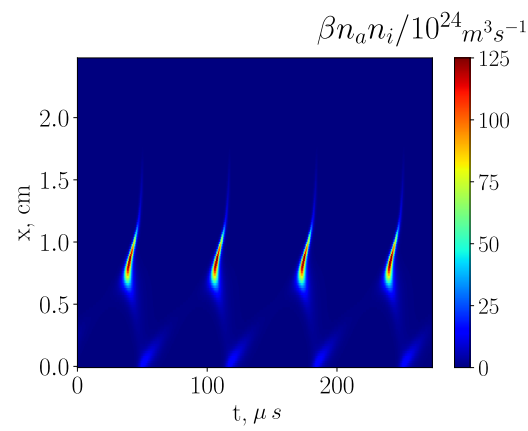

(h) Source (Reduced)

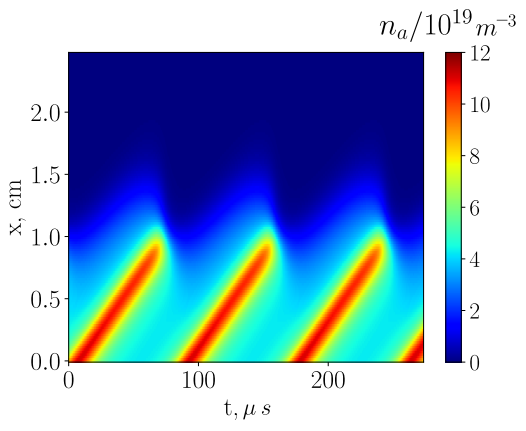

(c) $n_{a}$ (Reduced, lower $\beta$ )

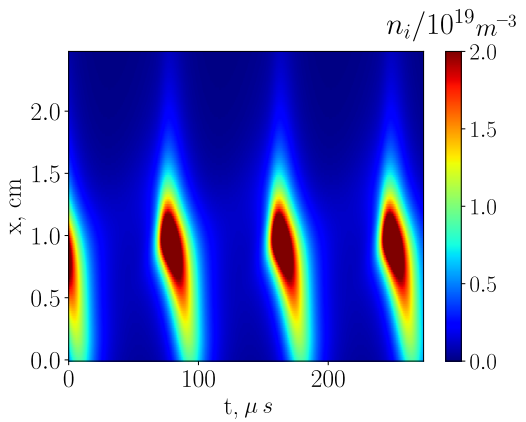

(f) $n_{i}$ (Reduced, lower $\beta$ )

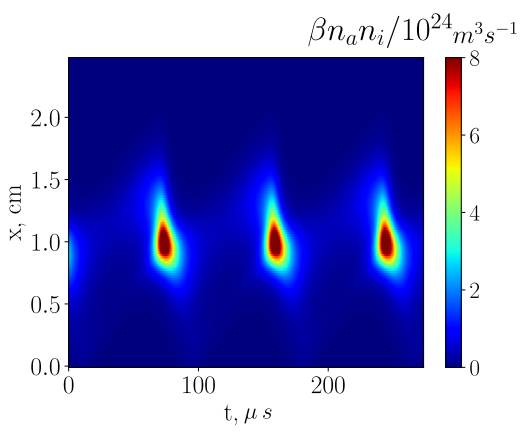

(i) Source (Reduced, lower $\beta$ )

Figure 10. Neutral density (a,b,c), ion density (d,e,f), and ionization source $\beta n_{i} n_{a}$ (g,h,i) for the full fluid model, the reduced model, and the reduced model with lower values of $\beta$ (see captions). Density values are normalized to $10^{19} \mathrm{~m}^{-3}$, and source values to $10^{24} \mathrm{~m}^{-3} \mathrm{~s}^{-1}$. The spatial domain is limited to channel region only, $2.5 \mathrm{~cm}$ from the anode.

To show that the atom velocity $v_{a}$ plays a defining role in the observed oscillation frequency, the full and reduced model (lower $\beta$ ) were compared against each other, Fig. 11. Both full and reduced (with lower $\beta$ ) model show approximately linear behaviour for the 
frequency, again suggesting the feedback loop mechanism supported by ion back-flow and advection of the neutral atoms. Interestingly, the ion current amplitudes are comparable between the full and reduced (lower $\beta$ ) models, Fig. 12 .

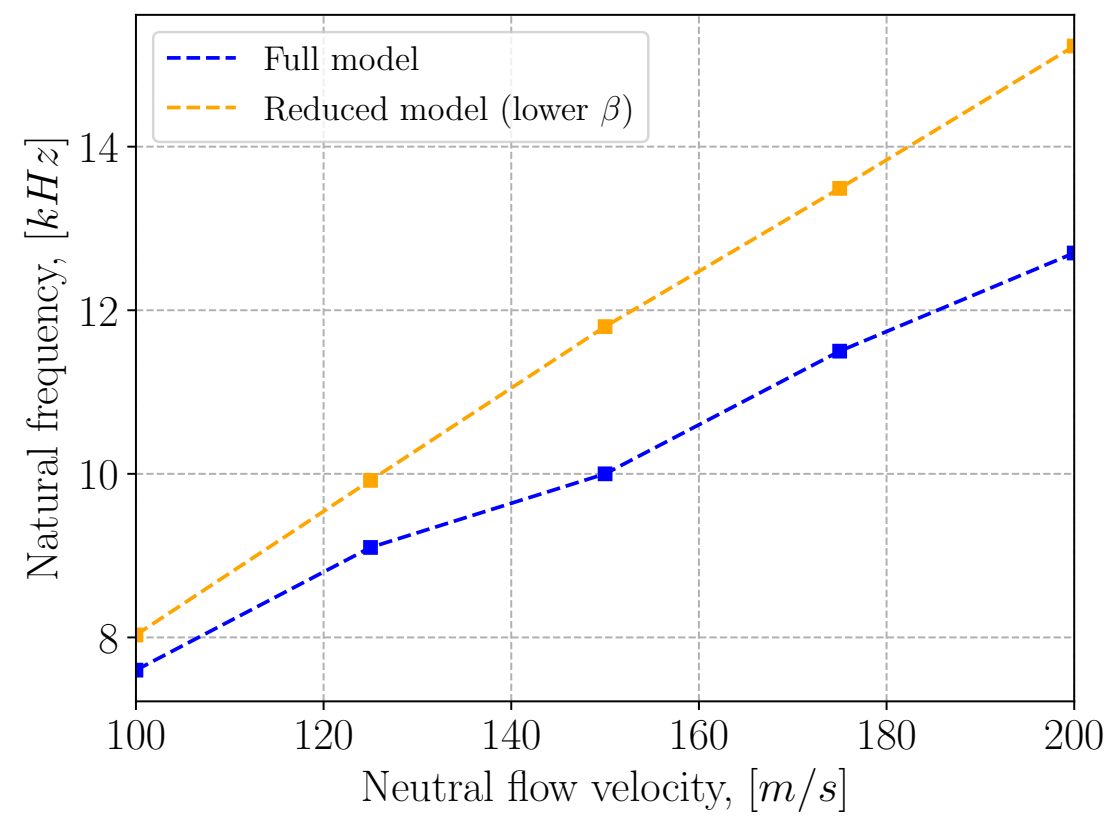

Figure 11. Oscillation frequency as a function of neutral velocity in full and reduced (with lower $\beta$ ) fluid models.

\section{SUMMARY}

In this paper, we have proposed a reduced continuum 1-D model with the ion velocity profile that has an ion back-flow (toward the anode) region. The model consists of two onedimensional equations for coupled dynamics of the ion and neutral densities. While the 0-D simplification of this model, commonly referred to as the predator-prey model, shows the oscillations, the continuum 1-D model with a uniform ion velocity and boundary conditions typical of Hall thruster show damped modes converging to the analytical stationary state. Under externally imposed perturbations at the left boundary, the 1-D continuum model shows limited convective amplification of the perturbation (in the region where the local value of the neutral density is smaller than plasma density) and subsequent convection (with a slow amplitude decay) where the ion and neutral density perturbations are mostly decoupled. Therefore the models with the uniform ion velocity, in the absence of additional 


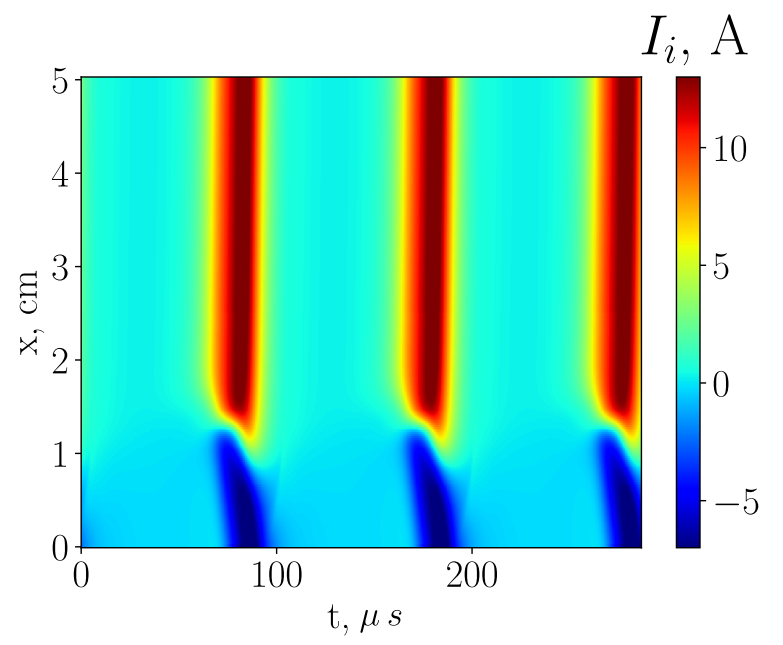

(a)

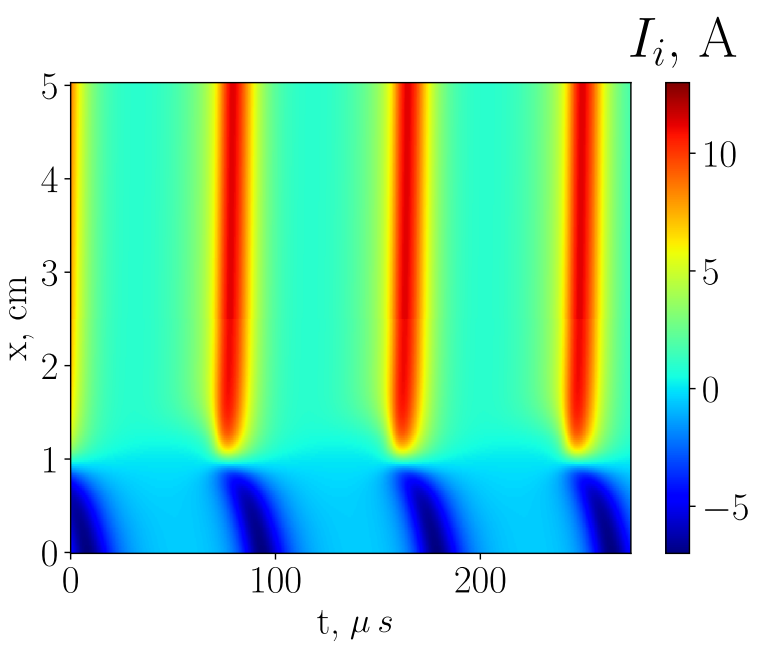

(b)

Figure 12. Temporal and spatial ion current dynamics in full model (a) and reduced model with lower $\beta$ (b), in Amperes (assuming cross section area $A=12.75 \pi \mathrm{cm}^{2}$ ).

effects e.g. such as temperature variations ${ }^{13}$, do not predict the excitation of the breathing modes.

The key element of the proposed continuum model is the ion velocity profile that has an ion back-flow (toward the anode) region. As it is shown here, such a system becomes unstable and shows self-consistent nonlinear oscillations. The comparison between this reduced model and the full self-consistent fluid model shows very similar characteristics for oscillations of the ion and neutral densities, and ion current. We have investigated the scaling of the frequency and amplitude of the oscillations as a function of the width of the back-flow region and neutral flow velocity which show generic $v_{a} / L_{b}$ dependence both in the reduced and full models. These results suggest that the presence of the ion back-flow region may be a critical condition for the breathing mode oscillations and the overlap of this region with the ionization region creates the closed feedback loop necessary for the instability. These results also indicate that the electron dynamics, in particular diffusion, which leads to the appearance of the back-flow region, is important for the breathing mode. A number of full models, reporting the breathing mode oscillations, include the electron diffusion either explicitly via the electron fluid equations, such as full fluid ${ }^{6}$ or hybrid ${ }^{33}$ formulations, or via full kinetic treatment as in the full kinetic (particle-in-cell) treatment ${ }^{39}$. The ion back-flow near the anode occurs as a result of the electron diffusion ensuring the current conservation 
to maintain quasineutrality. The ion stagnation in the back-flow region triggering enhanced ionization is suggested as the mechanism for the instability. It is also worth noting that the electron diffusion is also a reason for the appearance of the singular sonic point where the ion velocity is equal to the local sound velocity, $v_{i}=c_{s}$. The smooth (regular) solution is obtained when the sonic point is made regular by imposing some constraints on the current, mass flow rate, and plasma parameters. These constraints made singular point regular and

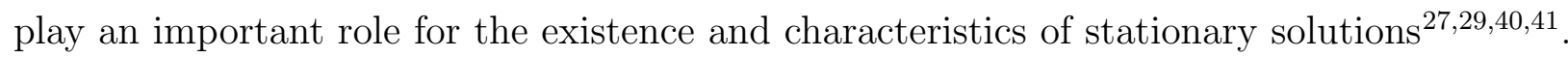
As it was shown in $\frac{18 \mid 42}{2}$ the constraints indeed define the stability of the resulting profile and characteristic frequency of the breathing mode.

One has to note, however, that low frequency ionization oscillations were also observed in the models without the electron diffusion, and hence, without back-flow region has been suggested ${ }^{9}$ that the high frequency oscillations due to the resistive axial modes $9 / 46 / 47$ is a driving mechanism for a breathing mode. Alternative mechanisms may be based on the temperature dependence of the ionization coefficient ${ }^{13}$, other electron temperature effects $\$ 48$, and/or more complex interactions between the low frequency modes involving ionization and higher frequency modes in the ion transit time frequency range as it was suggested earlier $\frac{18 / 49}{\text {. }}$ The simulations with the full model also demonstrate the existence of two different regimes of the breathing mode ${ }^{18}$, the so-called solo regime, where only the low frequency mode present, and the regime with coexisting low frequency and the high frequency ion transit time oscillations. The breathing mode oscillations reproduced by our reduced model are similar to the solo regime, but are different from the modes coexisting with high-frequency ion transit time oscillations. The existence and nature of the breathing mode together with the well pronounced high-frequency mode is an interesting question that will be addressed in future studies with the full model.

In summary, we would like to conclude that the importance of the reduced models is not only in their ability to predict reasonably well some essential features of the ionization modes in Hall thrusters but also, and perhaps, even more importantly, in pointing to some important physics involved in the modes excitation and characteristics. The reduced models typically involve a smaller number of adjustable parameters compared to the full models, e.g. as the values of the anomalous mobility are poorly known. Therefore, the tests of the properties and consequences of the reduced models in the experiments $\frac{15}{17 / 25}$ could be an effective approach to test the crucial physics responsible for the breathing modes oscillations. 


\section{ACKNOWLEDGMENTS}

This work is supported in part by US Air Force Office of Scientific Research FA955015-1-0226, NSERC Canada, and Compute Canada computational resources. The authors acknowledge with gratitude the important discussions with I. V. Romadanov, J. B. Simmonds, I.D. Kaganovich, and K. Hara.

\section{DATA AVAILABILITY}

The data that support the findings of this study are available from the corresponding author upon reasonable request.

\section{REFERENCES}

${ }^{1}$ V. Zhurin, J. Kahn, H. Kaufman, K. Kozubsky, and M. Day, "Dynamic characteristics of closed drift thrusters," in 23rd International Electric Propulsion Conference (1993).

${ }^{2}$ Y. Raitses and N. J. Fisch, "Parametric investigations of a nonconventional Hall thruster," Physics of Plasmas 8, 2579-2586 (2001).

${ }^{3}$ L. Wei, K. Han, C. Wang, H. Li, C. Zhang, and D. Yu, "Study on breathing mode oscillation suppression of self-excited Hall thrusters," Journal of Vacuum Science \& Technology A 30, 061304 (2012).

${ }^{4}$ A. Morozov and V. Savelyev, "Fundamentals of stationary plasma thruster theory," in Reviews of Plasma Physics (Springer, 2000) pp. 203-391.

${ }^{5}$ G. Hagelaar, J. Bareilles, L. Garrigues, and J.-P. Boeuf, "Two-dimensional model of a stationary plasma thruster," Journal of Applied Physics 91, 5592-5598 (2002).

${ }^{6} \mathrm{~S}$. Barral and E. Ahedo, "Low-frequency model of breathing oscillations in Hall discharges," Physical Review E 79, 046401 (2009).

${ }^{7}$ J. Boeuf and L. Garrigues, "Low frequency oscillations in a stationary plasma thruster," Journal of Applied Physics 84, 3541-3554 (1998).

${ }^{8}$ J. M. Fife, Hybrid-PIC modeling and electrostatic probe survey of Hall thrusters, Ph.D. thesis, Massachusetts Institute of Technology (1998).

${ }^{9} \mathrm{~S}$. Chable and F. Rogier, "Numerical investigation and modeling of stationary plasma thruster low frequency oscillations," Physics of Plasmas 12, 033504 (2005). 
${ }^{10} \mathrm{~K}$. Hara, I. Boyd, and V. Kolobov, "One-dimensional hybrid-direct kinetic simulation of the discharge plasma in a Hall thruster," Physics of Plasmas 19, 113508 (2012).

${ }^{11}$ J. Fife, M. Martinez-Sanchez, J. Szabo, J. Fife, M. Martinez-Sanchez, and J. Szabo, "A numerical study of low-frequency discharge oscillations in Hall thrusters," in 33rd Joint Propulsion Conference and Exhibit (2012) p. 3052.

${ }^{12}$ S. Barral and E. Ahedo, "On the origin of low frequency oscillations in Hall thrusters," AIP Conference Proceedings 993, 439-442 (2008).

${ }^{13}$ K. Hara, M. J. Sekerak, I. Boyd, and A. Gallimore, "Perturbation analysis of ionization oscillations in Hall effect thrusters," Physics of Plasmas 21, 122103 (2014).

${ }^{14}$ C. Wang, L. Wei, and D. Yu, "A Basic Predator-Prey Type Model for Low Frequency Discharge Oscillations in Hall Thrusters," Contributions to Plasma Physics 51, 981-988 (2011).

${ }^{15}$ E. Dale and B. Jorns, "Frequency scaling of the hall thruster breathing mode," in $A I A A$ Propulsion and Energy 2019 Forum (2019).

${ }^{16}$ E. Dale and B. Jorns, "Two-zone Hall thruster breathing mode mechanism, Part I: Theory," in 36th International Electric Propulsion Conference, University of Vienna, Austria (2019).

${ }^{17}$ E. Dale and B. Jorns, "Two-zone Hall thruster breathing mode mechanism, Part II: Experiment," in 36th International Electric Propulsion Conference, University of Vienna, Austria (2019).

${ }^{18}$ A. Smolyakov, O. Chapurin, I. Romadanov, Y. Raitses, and I. Kaganovich, "Theory and modelling of axial mode oscillations in Hall thruster," in AIAA Propulsion and Energy 2019 Forum.

${ }^{19}$ N. Yamamoto, T. Nakagawa, K. Komurasaki, and Y. Arakawa, "Discharge plasma fluctuations in Hall thrusters," Vacuum 65, 375-381 (2002).

${ }^{20}$ N. Yamamoto, K. Komurasaki, and Y. Arakawa, "Discharge current oscillation in Hall thrusters," Journal of Propulsion and Power 21, 870-876 (2005).

${ }^{21}$ N. Yamamoto, S. Yokota, K. Watanabe, A. Sasoh, K. Komurasaki, and Y. Arakawa, "Suppression of discharge current oscillations in a hall thruster," Transactions of the Japan Society for Aeronautical and Space Sciences 48, 169-174 (2005).

${ }^{22} \mathrm{~K}$. Hara and K. Kubota, "Direct kinetic simulation of ion acoustic turbulence in cathode plume," in 35th International Electric Propulsion Conference, Atlanta, Georgia, IEPC- 
2017-496 (2017).

${ }^{23}$ L. Wei, L. Han, D. Yu, and N. Guo, "Low-Frequency Oscillations in Hall Thrusters," Chinese Physics B 24, 055201 (2015).

${ }^{24}$ Y. Raitses, D. Staack, A. Dunaevsky, and N. J. Fisch, "Operation of a segmented Hall thruster with low-sputtering carbon-velvet electrodes," Journal of Applied Physics 99, 036103 (2006).

${ }^{25}$ I. Romadanov, Y. Raitses, and A. Smolyakov, "Hall thruster operation with externally driven breathing mode oscillations," Plasma Sources Science and Technology 27, 094006 (2018).

${ }^{26}$ N. J. Fisch, Y. Raitses, L. A. Dorf, and A. A. Litvak, "Variable operation of Hall thruster with multiple segmented electrodes," Journal of Applied Physics 89, 2040-2046 (2001).

${ }^{27}$ A. Cohen-Zur, A. Fruchtman, J. Ashkenazy, and A. Gany, "Analysis of the steady-state axial flow in the Hall thruster," Physics of Plasmas 9, 4363-4374 (2002).

${ }^{28}$ E. Ahedo and J. Rus, "Vanishing of the negative anode sheath in a Hall thruster," Journal of Applied Physics 98, 043306 (2005).

${ }^{29}$ L. Dorf, V. Semenov, and Y. Raitses, "Anode sheath in Hall thrusters," Applied Physics Letters 83, 2551-2553 (2003)

${ }^{30}$ L. Dorf, Y. Raitses, and N. J. Fisch, "Experimental studies of anode sheath phenomena in a Hall thruster discharge," Journal of Applied Physics 97, 103309 (2005).

${ }^{31}$ G. Hagelaar and L. Pitchford, "Solving the boltzmann equation to obtain electron transport coefficients and rate coefficients for fluid models," Plasma Sources Science and Technology 14, 722 (2005).

32 "SIGLO database," www.1xcat.net/SIGLO, [Retrived: June-2013].

${ }^{33}$ G. Hagelaar, J. Bareilles, L. Garrigues, and J.-P. Boeuf, "Modelling of stationary plasma thrusters," Contributions to Plasma Physics 44, 529-535 (2004).

${ }^{34}$ G. Hagelaar, J. Bareilles, L. Garrigues, and J.-P. Boeuf, "Role of anomalous electron transport in a stationary plasma thruster simulation," Journal of Applied Physics 93, 67-75 (2003).

${ }^{35} \mathrm{G}$. Hagelaar, "Modelling electron transport in magnetized low-temperature discharge plasmas," Plasma Sources Science and Technology 16, S57 (2007).

36 "Low temperAture magNetizeD plasMA benchmaRKs," https://www . landmark-plasma.com/ (2018), [Accessed: 26-March-2021]. 
37 "HALL Ion Sources simulation software," https://www.hallis-model.com/, [Accessed: 26-March-2021].

${ }^{38}$ L. Wei, C. Wang, K. Han, and D. Yu, "Effect of ionization distribution on the low frequency oscillations mode in Hall thrusters," Physics of Plasmas 19 (2012), $10.1063 / 1.3676160$.

${ }^{39} \mathrm{P}$. Coche and L. Garrigues, "A two-dimensional (azimuthal-axial) particle-in-cell model of a Hall thruster," Physics of Plasmas 21, 023503 (2014).

${ }^{40}$ E. Ahedo, P. Martinez-Cerezo, and M. Martinez-Sanchez, "One-dimensional model of the plasma flow in a Hall thruster," Physics of Plasmas 8, 3058-3068 (2001).

${ }^{41}$ K. Makowski, Z. Peradzynski, S. Barral, and M. A. Dudeck, "Review of the plasma fluid models in Stationary Plasma Thrusters," High Temperature Material Processes: An International Quarterly of High-Technology Plasma Processes 5 (2001).

${ }^{42}$ I. V. Romadanov, A. I. Smolyakov, E. A. Sorokina, V. V. Andreev, and N. A. Marusov, "Stability of ion flow and role of boundary conditions in a simplified model of the ExB plasma accelerator with a uniform electron mobility," Plasma Physics Reports 46, 363-373 $(2020)$.

${ }^{43}$ A. I. Morozov and V. V. Savel'ev, "One-dimensional hydrodynamic model of the atom and ion dynamics in a stationary plasma thruster," Plasma Physics Reports 26, 219-224 $(2000)$.

${ }^{44}$ A. I. Morozov and V. V. Savel'ev, "One-dimensional hybrid model of a stationary plasma thruster," Plasma Physics Reports 26, 875-880 (2000).

${ }^{45} \mathrm{~K}$. Hara and K. Hanquist, "Test cases for grid-based direct kinetic modeling of plasma flows," Plasma Sources Science \& Technology 27 (2018), 10.1088/1361-6595/aac6b9.

${ }^{46}$ E. Fernandez, M. K. Scharfe, C. A. Thomas, N. Gascon, and M. A. Cappelli, "Growth of resistive instabilities in exb plasma discharge simulations," Physics of Plasmas 15, 012102 (2008).

${ }^{47}$ O. Koshkarov, A. I. Smolyakov, I. V. Romadanov, O. Chapurin, M. V. Umansky, Y. Raitses, and I. D. Kaganovich, "Current flow instability and nonlinear structures in dissipative two-fluid plasmas," Physics of Plasmas 25, 011604 (2018).

${ }^{48}$ D. Staack, Y. Raitses, and N. J. Fisch, "Temperature gradient in Hall thrusters," Applied Physics Letters 84, 3028-3030 (2004). 
${ }^{49}$ J. Kurzyna, K. Makowski, Z. Peradzyński, A. Lazurenko, S. Mazouffre, and M. Dudeck, "Where is the breathing mode? High voltage Hall effect thruster studies with EMD method," AIP Conference Proceedings 993, 443-446 (2008). 